\title{
Generation of zonal flows by Rossby waves in the atmosphere
}

\author{
O. G. Onishchenko ${ }^{1}$, O. A. Pokhotelov ${ }^{1}$, R. Z. Sagdeev ${ }^{2}$, P. K. Shukla ${ }^{3}$, and L. Stenflo ${ }^{4}$ \\ ${ }^{1}$ Institute of Physics of the Earth, 123810 Moscow, 10 B. Gruzinskaya Str., Russia \\ ${ }^{2}$ Department of Physics, University of Maryland, College Park, Maryland 20 740, USA \\ ${ }^{3}$ Institut für Theoretische Physik IV, Fakultät für Physik und Astronomie, Ruhr-Univ. Bochum, D-44780 Bochum, Germany \\ ${ }^{4}$ Department of Plasma Physics, Umeå University S-90 187 Umeå, Sweden
}

Received: 26 June 2003 - Revised: 13 October 2003 - Accepted: 12 November 2003 - Published: 14 April 2004

Part of Special Issue "International Workshops on Nonlinear Waves and Chaos in Space Plasmas"

\begin{abstract}
A novel mechanism for the short-scale Rossby waves interacting with long-scale zonal flows in the Earth's atmosphere is studied. The model is based on the parametric excitation of convective cells by finite amplitude Rossby waves. We use a set of coupled equations describing the nonlinear interaction of Rossby waves and zonal flows which admits the excitation of zonal flows. The generation of such flows is due to the Reynolds stresses of the finite amplitude Rossby waves. It is found that the wave vector of the fastest growing mode is perpendicular to that of the pump Rossby wave. We calculate the maximum instability growth rate and deduce the optimal spatial dimensions of the zonal flows as well as their azimuthal propagation speed. A comparison with previous results is made. The present theory can be used for the interpretation of existing observations of Rossby type waves in the Earth's atmosphere.
\end{abstract}

\section{Introduction}

Generation of the zonal flows by Rossby waves is often observed in the Earth's atmosphere and in laboratory experiments (e.g. Petviashvili and Pokhotelov, 1992 and references therein). Zonal flows are a common feature of many planetary systems and are defined as zero-frequency modes propagating along the parallels. They vary on time scales slower than those corresponding to the Rossby waves. Zonal flows have for a long time been observed in experimental and numerical simulations of rotating neutral fluids, and have been invoked to explain the striped atmospheres of giant planets (Busse, 1994; Aubert et al., 2001, 2002; Cristensen, 2001). Regions of alternating azimuthal velocity have been found on Saturn and Jupiter by the Voyager spacecraft (Ingersoll, 1990). The zonal flows may play a crucial role in the evolution of the vortex structures that regulate the anomalous transport in planetary atmospheres (e.g. Kamenets et al.,

Correspondence to: P. K. Shukla

(profshukla@yahoo.de)
1996). They can arise in the Earth's atmosphere which frequently displays azimuthal streams.

In reality the planetary atmospheres can support both Rossby waves and zonal flows and they thus constitute a dynamical system which exhibits complex nonlinear interactions. Rossby waves are finite frequency dispersive waves propagating along the parallels in the azimuthal direction transverse to the beta plane where the inhomogeneities of the equilibrium atmosphere scale and the planetary rotation frequency play an important role. Since in the Earth's atmosphere the Rossby radius $r_{0}$ is large $(\simeq 2000 \mathrm{~km})$ and thus only a few times smaller then the radius of a planet, we focus our attention on perturbations of the atmosphere with spatial horizontal dimensions $\lambda_{\perp}=2 \pi / k_{\perp}$ comparable or smaller than $r_{0}$. In this approximation, the Rossby waves are described by the reduced dispersion relation

$\omega_{\boldsymbol{k}}=k_{x} u_{*} /\left(1+k_{\perp}^{2} r_{0}^{2}\right)$,

where $\omega_{\boldsymbol{k}}$ is the wave frequency, $\boldsymbol{k}$ is the wave vector, $k_{\perp}=\left(k_{x}^{2}+k_{y}^{2}\right)^{1 / 2}, k_{x(y)}$ is the $x(y)$-component of the wave vector, $r_{0}=\left(g H_{0}\right)^{1 / 2} / f$ is the Rossby radius, $g$ is the gravitational acceleration, $f$ is the Coriolis frequency which depends on $y, u_{*}=g \partial_{y}\left(H_{0} / f\right)$ is the Rossby speed, and $H_{0}$ stands for the atmospheric reduced height. For the sake of convenience we consider a shallow rotating atmosphere, assuming that the wave motions are localized in the vicinity of a given latitude $\lambda=\lambda_{0}$. Similar to Kaladze et al. (2003) we introduce a two-dimensional local Cartesian system of coordinates $(x, y)$ with longitudinal, $x=\varphi R \cos \lambda_{0}$, and latitudinal, $y=\left(\lambda-\lambda_{0}\right) R$, coordinates. In this coordinate system the $x$-axis is directed from the west to the east and the $y$-axis points from the south to the north. Furthermore, $\varphi$ is the latitude and $R$ is distance from the Earth's center.

We note that the dispersion relation (1) resembles the drift wave dispersion relation (e.g. Hasegawa and Mima, 1978; Hasegawa et al., 1979) for a nonuniform magnetoplasma where the atmospheric reduced height, the Rossby speed and the Rossby radius are replaced by the drift wave potential, the 
electron diamagnetic drift velocity and the ion gyroradius at the electron temperature, respectively. The Coriolis force in fluids is similarly replaced by the ion Lorentz force. However, electrostatic drift waves are pseudo-three-dimensional contrary to flute-like Rossby waves. Both Rossby and drift waves are obtained in the geostrophic or drift approximations where the characteristic wave frequency is much smaller than the Coriolis or the ion gyrofrequency. Clearly, there are similarities between Rossby and drift waves, despite the fact that the two waves appear under different physical conditions. The dynamics of nonlinear Rossby and drift waves is governed by the Charney (1948) and Hasegawa-Mima (1978) equations, in which the nonlinearity comes from the fluid advection and the nonlinear ion polarization drifts, respectively.

Recently, there has been renewed interest in examining the nonlinear coupling between coherent and incoherent drift waves and zonal flows (or convective cells) in nonuniform magnetoplasmas (e.g. Smolyakov et al., 2000; Manfredi et al., 2001; Shukla and Stenflo, 2002). It has been found that pseudo-three dimensional drift waves strongly couple with zonal flows whose dynamics is governed by the drift wave stresses driven Navier-Stokes equation. Thus, the latter is nonlinearly coupled with the Hasegawa-Mima equation in the drift wave-zonal flow theory. On the other it has been shown (Shukla and Stenflo, 2003) that the zonal flow generation in fluids can be considered within a simple model of Rossby-wave turbulence described by the two-dimensional Charney equation (Charney, 1948; Pedlosky, 1987). In the short wavelength limit, viz. $k_{\perp} r_{0} \gg 1$, the latter is

$r_{0}^{2} \nabla_{\perp}^{2} \partial_{t} h-u_{*} \partial_{x} h+f r_{0}^{4}\left\{h, \nabla_{\perp}^{2} h\right\}=0$.

In this model the atmosphere is treated as an incompressible shallow water fluid of depth $H=H_{0}(1+h)$, where $H_{0}$ is the unperturbed depth which may depend weakly on the coordinate $y$ and $h$ stands for the dimensionless wave amplitude. Furthermore, $\{A, B\} \equiv\left(\partial_{x} A\right) \partial_{y} B-\left(\partial_{y} A\right) \partial_{x} B$ denotes the Poisson bracket.

Since the zonal flow varies on a much longer time scale than the small-scale Rossby waves one can use a multiple scale expansion, assuming that there is a sufficient spectral gap separating the large- and small-scale motions. Following the standard procedure to describe the evolution of the coupled system (Rossby waves plus zonal flows), we decompose the perturbation of the atmospheric dimensionless depth $h$ into its low- and high-frequency parts, that is $h=\hat{h}+\tilde{h}$, where $\hat{h}(y, t)$ refers to the large-scale zonal flow and $\tilde{h}(\boldsymbol{r}, t)$ to the short-scale Rossby wave. Averaging Eq. (2) over the fast short scales, we obtain the evolution equation for the mean flow

$\nabla_{\perp}^{2} \partial_{t} \hat{h}=-f r_{0}^{2} \overline{\left\{\tilde{h}, \nabla_{\perp}^{2} \tilde{h}\right\}}$,

where the bar denotes the averaging process.

In Eq. (3) the term on the right-hand side describes the Reynolds stresses induced by the short-scale Rossby waves. The nonlinear coupling of these waves with the zonal flow is governed by

$r_{0}^{2} \nabla_{\perp}^{2} \partial_{t} \tilde{h}-u_{*} \partial_{x} \tilde{h}=-f r_{0}^{4}\left(\left\{\tilde{h}, \nabla_{\perp}^{2} \hat{h}\right\}+\left\{\hat{h}, \nabla_{\perp}^{2} \tilde{h}\right\}\right)$.
The Rossby waves are considered as a superposition of the pump wave and two sidebands, that is $\tilde{h}=h_{0}+\tilde{h}_{+}+\tilde{h}_{-}$, where for the pump wave we have $h_{0}=h_{\boldsymbol{k}} \exp \left(i\left(\boldsymbol{k} \cdot \boldsymbol{r}-\omega_{\boldsymbol{k}} t\right)\right)+h_{\boldsymbol{k}}^{*} \exp \left(-i\left(\boldsymbol{k} \cdot \boldsymbol{r}-\omega_{\boldsymbol{k}} t\right)\right)$ with the frequency $\omega_{\boldsymbol{k}}$ given by Eq. (1).

The change in the zonal flow amplitude is given by $\hat{h}=h_{\boldsymbol{q}} \exp (i(\boldsymbol{q} \cdot \boldsymbol{r}-\Omega t))+h_{\boldsymbol{q}}^{*} \exp (-i(\boldsymbol{q} \cdot \boldsymbol{r}-\Omega t))$, where $\boldsymbol{q}=q \quad \hat{\boldsymbol{y}}$ and $\hat{\boldsymbol{y}}$ is the unit vector along the latitude. For the Rossby side-bands we have $\tilde{h}_{ \pm}=h_{\boldsymbol{k}_{ \pm}} \exp \left(i\left(\boldsymbol{k}_{ \pm} \cdot \boldsymbol{r}-\omega_{\boldsymbol{k}_{ \pm}} t\right)\right)+h_{\boldsymbol{k}_{ \pm}}^{*} \exp \left(-i\left(\boldsymbol{k}_{ \pm} \cdot \boldsymbol{r}-\omega_{\boldsymbol{k}_{ \pm}} t\right)\right)$, where $\omega_{\boldsymbol{k}_{ \pm}}=\omega_{\boldsymbol{k}} \pm \Omega$ and $\boldsymbol{k}_{ \pm}=\boldsymbol{k} \pm \boldsymbol{q}$ are the frequencies and wave vectors of the Rossby sidebands.

Substituting $h_{0}, \hat{h}$ and $\tilde{h}_{ \pm}$into Eq. (3), we obtain

$$
\begin{aligned}
\Omega \hat{h}_{\boldsymbol{q}}=- & i f r_{0}^{2} \frac{(\boldsymbol{k} \times \boldsymbol{q})_{z}}{q^{2}} \\
& \cdot\left(\left(\boldsymbol{k}_{+}^{2}-\boldsymbol{k}^{2}\right) h_{\boldsymbol{k}_{+}} h_{\boldsymbol{k}}^{*}-\left(\boldsymbol{k}_{-}^{2}-\boldsymbol{k}^{2}\right) h_{\boldsymbol{k}_{-}}^{*} h_{\boldsymbol{k}}\right) .
\end{aligned}
$$

where the expressions for Fourier amplitudes $h_{\boldsymbol{k}_{+}}$and $h_{\boldsymbol{k}_{-}}^{*}$, found from Eq. (4), are

$$
\begin{aligned}
& h_{\boldsymbol{k}_{+}}=-i \frac{f r_{0}^{2}(\boldsymbol{k} \times \boldsymbol{q})_{z}}{\Omega+\delta \omega_{+}} \frac{k^{2}-q^{2}}{k_{+}^{2}} \hat{h}_{\boldsymbol{q}} h_{\boldsymbol{k}}, \\
& h_{\boldsymbol{k}_{-}}^{*}=i \frac{f r_{0}^{2}(\boldsymbol{k} \times \boldsymbol{q})_{z}}{\Omega-\delta \omega_{-}} \frac{k^{2}-q^{2}}{k_{-}^{2}} \hat{h}_{\boldsymbol{q}} h_{\boldsymbol{k}}^{*},
\end{aligned}
$$

where $\delta \omega_{ \pm} \equiv \omega_{\boldsymbol{k}}-k_{x} u_{*} / r_{0}^{2} \boldsymbol{k}_{ \pm}^{2}$. Substituting Eqs. (6) and (7) into Eq. (5) we have (cf. Shukla and Stenflo, 2003)

$$
\begin{aligned}
\Omega= & -f^{2} \frac{(\boldsymbol{k} \times \boldsymbol{q})_{z}^{2}\left(k^{2}-q^{2}\right) r_{0}^{4}\left|h_{0}\right|^{2}}{q^{2}} \\
& \cdot\left(\frac{\left(\boldsymbol{k}_{+}^{2}-\boldsymbol{k}^{2}\right)}{\boldsymbol{k}_{+}^{2}} \frac{1}{\Omega+\delta \omega_{+}}+\frac{\left(\boldsymbol{k}_{-}^{2}-\boldsymbol{k}^{2}\right)}{\boldsymbol{k}_{-}^{2}} \frac{1}{\Omega-\delta \omega_{-}}\right) .
\end{aligned}
$$

The dispersion relation (8) is in general too cumbersome for analysis, and it can thus, only be solved numerically. In order to simplify it we consider the case of most interest $q \ll k$, when the typical scales of the zonal flows are much larger then the scales of the Rossby waves. In this limiting case we have the following expansions

$$
\begin{aligned}
& \delta \omega_{ \pm} \simeq \mp \boldsymbol{q} \cdot \boldsymbol{v}_{g}-\frac{q^{2} v_{g}^{\prime}}{2}, \\
& \begin{aligned}
\frac{\left(\boldsymbol{k}_{+}^{2}-\boldsymbol{k}^{2}\right)}{\boldsymbol{k}_{+}^{2}} & \frac{1}{\Omega+\delta \omega_{+}}+\frac{\left(\boldsymbol{k}_{-}^{2}-\boldsymbol{k}^{2}\right)}{\boldsymbol{k}_{-}^{2}} \frac{1}{\Omega-\delta \omega_{-}} \\
& \simeq-\frac{q^{2} \Omega v_{g}^{\prime}}{\omega_{\boldsymbol{k}}\left(\left(\Omega-\boldsymbol{q} \cdot v_{g}\right)^{2}-\left(v_{g}^{\prime} q^{2} / 2\right)^{2}\right)},
\end{aligned}
\end{aligned}
$$

where

$v_{g}^{\prime} \equiv \frac{\partial v_{g}}{\partial k_{y}}=\frac{\partial^{2} \omega_{\boldsymbol{k}}}{\partial k_{y}^{2}}=-\omega_{\boldsymbol{k}} \frac{2\left(k_{x}^{2}-3 k_{y}^{2}\right)}{k^{4}}$.

Here $\quad v_{g} \equiv \partial \omega_{k} / \partial k_{y}=-2 k_{y} \omega_{k} / k^{2}$ is the latitudinal (y-component) pump Rossby group velocity. We note 
that $v_{g}^{\prime}$ can change sign when $k_{x}= \pm 3^{\frac{1}{2}} k_{y}$. This occurs on the Rossby wave caustics. Substituting Eqs. (9)-(11) into Eq. (8), we obtain the dispersion relation

$$
\begin{aligned}
\Omega_{ \pm} \simeq \boldsymbol{q} \cdot \boldsymbol{v}_{g} \pm i \\
\quad \cdot\left(-v_{g}^{\prime} \frac{(\boldsymbol{k} \times \boldsymbol{q})_{z}^{2} f^{2} r_{0}^{4} k^{2}\left|h_{0}\right|^{2}}{\omega_{\boldsymbol{k}}}-\left(\frac{v_{g}^{\prime} q^{2}}{2}\right)^{2}\right)^{1 / 2}
\end{aligned}
$$

If $v_{g}^{\prime} \omega_{k}^{-1}<0$, then the upper sign in Eq. (12) yields an instability. This condition is similar to the Lighthill criterion for modulation instability in nonlinear optics (Lighthill, 1965).

According to Eqs. (11) and (12) for a given $k_{x}\left(k_{x}>0\right)$ the instability condition applies to Rossby pump waves with wave vectors localized in the cone $k_{x} / 3^{\frac{1}{2}}>k_{y}>-k_{x} / 3^{\frac{1}{2}}$. The maximum growth is attained at the axis of the cone when $k_{y}=0$. In this case the mode is purely growing with the growth rate

$\gamma=-i \Omega_{+}=\left(2 f^{2} q^{2} k^{2} r_{0}^{4}\left|h_{0}\right|^{2}-\frac{q^{4}}{k^{4}} \omega_{k}^{2}\right)^{1 / 2}$.

Expression (13) describes the initial (linear) stage of zonal flow growth due to the parametric instability of the shortscale Rossby waves.

It follows from Eq. (13) that the wavenumbers $q$ of the growing modes are localized in the range

$0<\left(\frac{q}{k}\right)_{\max }^{2}<2\left(\frac{f}{\omega_{k}}\right)^{2}\left(k r_{0}\right)^{4}\left|h_{0}\right|^{2}$

For fixed $k$, the fastest wave growth is attained at the value of $(q / k)^{2}$ given by

$\left(\frac{q}{k}\right)_{\max }^{2}=\left(\frac{f}{\omega_{k}}\right)^{2}\left(k r_{0}\right)^{4}\left|h_{0}\right|^{2}$

This maximum growth rate is thus

$\gamma_{\max }=\frac{f^{2}}{k\left|u_{*}\right|}\left(k r_{0}\right)^{6}\left|h_{0}\right|^{2}$,

which shows that $\gamma_{\max }$ increases as $k^{5}$ in the short wavelength limit $\left(k r_{0} \gg 1\right)$. Physically, this instability is a manifestation of an inverse cascade. It shows that the spectral energy of the short-scale Rossby wave turbulence is transferred into the long scales of the zonal flows, i.e. the Rossby wave energy is converted into the energy of slow zonal motions. The transport of energy from short- to large scales is in twodimensional turbulence theory consistent with conservation of the total energy and enstrophy (Charney, 1948).

As follows from our analysis, the small-scale Rossby waves in a shallow rotating atmosphere are unstable with respect to the long wavelength perturbations. These perturbations are accompanied by the excitation of long wavelength modes with the velocity $v_{z f}=f r_{0}^{2} q\left|h_{0}\right|$, i.e. zonal flows. Replacing $q$ by its optimum value from Eq. (15) we obtain

$v_{z f}=\frac{\left(f r_{0}\right)^{2}}{\left|u_{*}\right|}\left(k r_{0}\right)^{4}\left|h_{0}\right|^{2}$.
The peculiar feature of this instability is that it appears solely for Rossby waves that are localized in the cone bounded by the caustics for which $v_{g}^{\prime}=0$. This can lead to the formation of a so-called caustic shadow in the spectrum of the Rossby waves.

For typical parameters of the Earth's atmosphere $f \approx 1.6 \times 10^{-4} \mathrm{~s}^{-1}, r_{0} \approx 2 \times 10^{6} \mathrm{~m}, h_{0} \simeq 10^{-2}, k r_{0} \simeq 5-10$ and $\left|u_{*}\right| \simeq 3 \times 10^{2} \mathrm{~m} / \mathrm{s}$ we obtain $\gamma_{\max } \simeq\left(2 \times 10^{-5}-8 \times 10^{-4}\right) \mathrm{s}^{-1}$, $\lambda_{\max }=2 \pi / q_{\max } \simeq(110-1700) \mathrm{km}$ and $v_{z f}=(22-350) \mathrm{m} / \mathrm{s}$. These estimates are consistent with existing observations. Thus, it is possible that the parametric instability of Rossby waves is responsible for the generation of mean flows in the atmospheres of rotating planets.

The parametric instability investigated in the present paper is generic to a wide class of similar instabilities in space and laboratory plasmas. On one hand it can play a role in the Earth's ionosphere where it results in the formation of a turbulent Alfvén boundary layer (Pokhotelov et al., 2003). On the other hand, the parametric instability can give rise to the generation of shear flows in laboratory plasmas where it may substantially influence the plasma drift turbulence suppressing the transport coefficients (e.g. Smolyakov et al., 2000; Manfredi et al., 2001). We note that the expression for the instability growth rate obtained by Smolyakov et al. (2000) and Manfredi et al. (2001) is similar to that of Eq. (13) except for the second term in the square root. In the mathematical formalism (the wave equation in the eikonal approximation based on the Hasegawa-Mima model (Hasegawa and Mima, 1978; Hasegawa et al., 1979) of Smolyakov et al. (2000) this additional term corresponds to wave dispersion spreading which was unfortunately neglected in both these papers. However, our analysis presented above shows that by taking this additional effect into consideration we can treat the case of main interest, that is, we can consider the fastest growing mode. This makes it possible to obtain the expression for the maximum instability growth rate and to define the optimal parameters of the zonal flows.

In the present study we have demonstrated how zonal flows in a shallow rotating atmosphere can be excited by finite amplitude Rossby waves. The driving mechanism of this instability is due to the Reynolds stresses which are inevitably inherent for finite amplitude short scale Rossby waves. Hence, our investigation provides an essential nonlinear mechanism for the transfer of spectral energy from short scale Rossby waves to long scale enhanced zonal flows in the Earth's atmosphere. We note that finite amplitude Rossby waves can be nonlinearly coupled to other atmospheric modes, e.g. to large amplitude long wavelength inertial waves (Falkovich, 1992; Pokhotelov et al., 1995). These authors considered a scenario when the inertial sidebands interact with the inertial pump wave and produce a low-frequency advection force, which becomes a driver for Rossby type perturbations. 
Acknowledgements. This research was partially supported by the Commission of the EU (Grant No. INTAS-01-0456), by the Russian Fund for Basic Research through the Grant No. 02-05-64 612 and by the Russian Academy of Sciences through the Grant "Physics of the Atmosphere: electrical processes and radiophysical methods". Two of us (O. A. P. and R. Z. S.) are grateful for the financial support provided by the International Space Science Institute, Bern, Switzerland, within the framework of the project "Identification of Nonlinear Processes in Space Data".

Edited by: G. S. Lakhina

Reviewed by: two referees

\section{References}

Aubert, J., Brito, D., Cardin, P., Nataf, H.-C., and Masson, J.-P.: A systematic experimental study of spherical shell rotating convection in water and liquid gallium, Phys. Earth Planet. Int., 128, 51, 2001.

Aubert, J., Jung, S., and Swinney, H. L.: Observations of zonal flow created by potential vorticity mixing in a rotating fluid, Geophys.Res. Lett., 29, dpi: 10.1029/2002GLO15 422, 2002.

Busse, F. H.: Convection driven zonal flows and vortices in themajor planets, Chaos, 4, 123, 1994.

Charney, J. G.: On the scale of atmospheric motions, Publ. Kosjones. Nors. Videnshap. Akad. Oslo, 17, 3, 1948.

Christensen, U. R.: Zonal flow driven by deep convection in themajor planets, Geophys. Res. Lett., 13, 2553, 2001.

Falkovich, G.: Inverse cascade and wave condensate in mesoscale atmospheric turbulence, Phys. Rev. Lett., 69, 3173, 1992.

Hasegawa, A. and Mima, K.: Pseudo-three-dimensional turbulence in magnetized uniform plasma, Phys. Fluids, 21, 87, 1978.

Hasegawa, A., Maclennan, C., and Kodama, Y.: Nonlinear behaviour and turbulence spectra of drift waves and Rossby waves, Phys. Fluids, 22, 2122, 1979.
Ingersoll, A.: Atmospheric dynamics of the outer planets, Science, 248, 308, 1990.

Kaladze, T. D., Pokhotelov, O. A., Sagdeev, R. Z., Stenflo, L., and Shukla, P. K.: Planetary electromagnetic waves in the ionospheric E-layer, J. Atmos. Solar-Terr. Phys., 65, 757, 2003.

Kamenets, F. F., Korobov, I. I., and Onishchenko, O. G.: Evolution of vortices forming in Jupiter's atmosphere after the collision of the planet with Comet Shoemaker-Levy 9, JETP Lett., 64, 350, 1996.

Lighthill, M. J.: Group velocity, J. Inst. Maths. Applics, 1, 1, 1965.

Manfredi, G., Roch, C. M., and Dendy, R. O.: Zonal flow and streamergeneration in drift turbulence, Plasma Phys. Control. Fusion, 43, 825, 2001.

Pedlosky, J.: Geophysical Fluid Dynamics, Springer-New York, 1987.

Petviashvili, V. I. and Pokhotelov, O. A.: Solitary Waves in Plasmas and in the Atmosphere, Gordon and Breach, ReadingPhiladelphia, 1992.

Pokhotelov, O. A., McKenzie, J. F., Shukla, P. K., and Stenflo, L.: Nonlinearly coupled inertial and Rossby waves, Phys. Fluids, 7, $1785,1995$.

Pokhotelov, O. A., Onishchenko, O. G., Sagdeev, R. Z., and Treumann, R. A.: Nonlinear dynamics of the inertial Alfvén waves in the Earth's ionosphere. Generation of convective cells, J. Geophys. Res., 108, 1291, doi: 10.1029/2003JA00 9888, 2003.

Shukla, P. K. and Stenflo, L.: Nonlinear interaction between driftwaves and zonal flows, Eur. Phys. J. D, 20, 103, 2002.

Shukla, P. K. and Stenflo, L.: Generation of zonal flows by Rossby waves, Phys. Lett. A, 307, 154, 2003.

Smolyakov, A. I., Diamond, P. H., and Shevchenko, V. I.: Zonal flowgeneration by parametric instability in magnetized plasmas and geostrophicfluids, Phys. Plasmas, 7, 1349, 2000. 